\title{
A Continuous-Time Model of Bilateral Bargaining
}

\author{
Juan Ortner* \\ Boston University
}

December 13, 2018

\begin{abstract}
This paper constructs a continuous-time model of bilateral bargaining to study how fluctuations in bargaining power affect the outcomes of negotiations. The paper deals with the technical complexities that arise when modeling games in continuous time by building strategy restrictions into the equilibrium definition. These restrictions select a unique equilibrium, which is characterized by a system of ordinary differential equations. This unique equilibrium corresponds to the limiting subgame perfect equilibrium of discrete-time bargaining games with frequent offers.

JEL CLASSIFICATION CODES: C73, C78.
\end{abstract}

KEYWORDS: bargaining, continuous-time games, time-varying bargaining power.

${ }^{*}$ I am indebted to Sylvain Chassang, Faruk Gul, Bart Lipman and Wolfgang Pesendorfer for advice. E-mail: jortner@bu.edu. 


\section{Introduction}

In many real life negotiations the relative strength of the parties changes over time. While one side might start bargaining from a strong position, bargaining power might change hands as the negotiation proceeds. For instance, in wage negotiations the relative bargaining power of firm and workers depends on the unemployment rate, a variable that fluctuates along with the economic cycle. In legislative bargaining the ability of a political party to implement its preferred policy depends on the number of seats it controls in Congress, a quantity that moves over time together with the party's political power and popularity. In mergers and acquisitions the way in which the firms divide the gains from joint operations may also depend on time-varying variables, such as market valuations and the general economic environment.

This paper introduces a model of bilateral bargaining to study how time-varying bar-

gaining power affects negotiation outcomes. The model's key variable is an exogenous and publicly observable diffusion process, which measures the player's relative bargaining power, and whose realization at each moment in time determines the identity of the player with proposal power. The game is played in continuous time, and at each instant prior to agreement the player with proposal power can make an offer to her opponent. There is no deadline, and bargaining continues indefinitely until an offer is accepted.

I show that this continuous-time model has a unique equilibrium, which is characterized by a system of ordinary differential equations. This tractable equilibrium characterization allows me to study how different features of the environment affect bargaining outcomes. For instance, I derive conditions under which more uncertainty about future bargaining power benefits the weaker player. The tractability of the model makes it amenable to a variety of extensions. I illustrate this by presenting an application of the model to study political negotiations with supermajority rules.

There are technical difficulties that arise when modeling games with observable actions in continuous time (e.g. Simon and Stinchcombe (1989), Bergin and MacLeod (1993)). The 
key difficulties are defining proper sub-games, and guaranteeing that a given strategy profile gives rise to a unique path of play. Simon and Stinchcombe (1989) deal with these difficulties by restricting players to take actions in discrete grids, and then taking limits as the grid size goes to zero. Bergin and MacLeod (1993) introduce inertia in strategies.

When analyzing continuous-time bargaining games, a related difficulty is that subgame perfection doesn't refine the set of equilibria as it does when the game is played in discrete time. In discrete-time bargaining games the responder incurs a fixed cost of delay if she doesn't accept the current offer, since she must wait at least one time period to reach an agreement. This fixed cost of delay imposes restrictions on the strategies that players use in a subgame perfect equilibrium (SPE). In contrast, the responder does not face a fixed cost of delay when the game is in continuous time, since she can accept a new offer within an arbitrarily short time period. This leads to a multiplicity of equilibrium outcomes. This multiplicity of outcomes was previously noted by Bergin and MacLeod (1993), who show that any division of the surplus can be supported as an equilibrium outcome in a bargaining game in which players can take actions in continuous time. ${ }^{1}$

This paper deals with this multiplicity by building into the definition of equilibrium of the continuous-time game the intuitive restriction that the responder always accepts offers equal to her continuation payoff of waiting until she becomes proposer. I show that this additional restriction selects a unique equilibrium. Moreover, this unique equilibrium corresponds to the (also unique) limiting SPE of the discrete-time version of the bargaining game, when players can make offers arbitrarily frequently. Hence, the tractable equilibrium of the continuoustime game provides a good approximation of settings with small bargaining frictions.

The present paper relates to the literature on bargaining games in continuous time. Perry and Reny (1993) construct a continuous-time bilateral bargaining game in which players can strategically time their offers (see also Sakovics (1993)). Ambrus and Lu (2015) study

\footnotetext{
${ }^{1}$ As noted above, Bergin and MacLeod (1993) get around this issue by introducing inertia in the players' strategies. Perry and Reny (1993) also note this multiplicity of equilibrium outcomes in continuous time bargaining games, and also deal with this issue by placing restrictions on the timing of offers/counteroffers.
} 
a continuous-time coalitional bargaining game with a fixed deadline in which players get random opportunities to make proposals through a Poisson process. A common feature of these models is the presence of restrictions on the timing of offers and counteroffers. In Perry and Reny (1993) these restrictions appear in the form of waiting times and reaction times; i.e., players cannot make a new offer immediately after making a proposal, and must wait a positive amount of time before replying to an offer. In Ambrus and Lu (2015) the restrictions are at the heart of the model, since players can only make proposals when the Poisson process hits. These restrictions allow these authors to sidestep the technical issues that arise when modeling bargaining games in continuous time, and bring the analysis closer to that of discrete-time bargaining games. In contrast, the current model features no restrictions on the timing of offers (besides the identity of the proposer), and proposes a novel way of dealing with the technical difficulties inherent to continuous time games.

More recently, Ortner (2017a), Daley and Green (2018) and Villamizar (2018) study continuous-time bargaining games with one-sided incomplete information. They deal with the technical difficulties of continuous-time bargaining games by imposing indifference restrictions on equilibria that are very much in the same spirit as the restrictions I impose in the current paper. The current paper establishes the validity of this approach (within a particular environment) by showing that these restrictions select the (unique) subgame perfect equilibrium outcome of discrete-time bargaining games with frequent offers.

Finally, Simsek and Yildiz (2014) also study a bilateral bargaining game in which the players' bargaining power evolves stochastically over time. They study settings in which players have optimistic beliefs about their future bargaining power, and show that optimism can give rise to inefficient delays if players expect bargaining power to become more durable in the future. Simsek and Yildiz (2014) analyze a discrete-time bargaining game, and focus on the properties of the game's limiting SPE with frequent offers. 


\section{Model}

Players and preferences. Two players, $i=1,2$, bargain over how to divide a perfectly divisible surplus of size 1 ; the set of possible agreements is $\left\{y \in \mathbb{R}_{+}^{2}: y_{1}+y_{2}=1\right\}$. Time is continuous and players can reach an agreement at any time $t \in[0, \infty)$. Both players are risk neutral expected utility maximizers and discount future payoffs at the common rate $r>0$. The payoff that player $i$ gets from receiving a (possibly random) share $z \in[0,1]$ of the surplus at a (possibly random) time $\tau$ is $\mathbb{E}\left[e^{-r \tau} z\right]$. If players fail to reach an agreement in finite time they both get a payoff of zero.

Relative bargaining power and bargaining protocol. The key variable of the model is a publicly observable stochastic process, which measures the players' relative bargaining power and which I denote by $x_{t}$. Let $B=\left\{B_{t}, \mathcal{F}_{t}: 0 \leq t<\infty\right\}$ be standard Brownian motion on the probability space $(\Omega, \mathcal{F}, \mathbf{P}) .^{2}$ Process $x_{t}$ evolves as a Brownian motion with constant drift $\mu$ and constant volatility $\sigma>0$, with reflecting boundaries at -1 and 1 :

$$
d x_{t}=\mu d t+\sigma d B_{t}
$$

if $x_{t} \in(-1,1)$; if $x_{t}$ reaches either -1 or 1 it reflects back. ${ }^{3}$

At each time $t \in[0, \infty)$ before players reach an agreement, the value of $x_{t}$ determines the identity of the proposer: player 1 makes offers at $t$ if $x_{t} \geq 0$ and player 2 makes offers if $x_{t}<0$. Suppose for instance that $x_{0} \geq 0$. In this case player 1 is proposer from time 0 until time $\tau_{1}:=\inf \left\{t \geq 0: x_{t}<0\right\}{ }^{4}$ At any moment until $\tau_{1}$, player 1 can make an offer $z \in\left\{y \in \mathbb{R}_{+}^{2}: y_{1}+y_{2}=1\right\}$ to player 2. There are no restrictions on the number or timing of offers that player 1 can make between $t=0$ and $\tau_{1}$. If player 2 accepts an offer before $\tau_{1}$ the

\footnotetext{
${ }^{2}$ Throughout the paper, the filtration $\mathcal{F}_{t}$ is assumed to be right-continuous: for all $t \geq 0, \mathcal{F}_{t}=\mathcal{F}_{t^{+}}:=$ $\cap_{\varepsilon>0} \mathcal{F}_{t+\varepsilon}$.

${ }^{3}$ In Ortner (2017b) I study a political bargaining game in which the parties' popularity evolves as a reflecting Brownian motion. See Harrison (1985) for a detailed analysis of diffusion processes with reflecting boundaries.

${ }^{4}$ Note that time $\tau_{1}$ is a stopping time, since the filtration $\mathcal{F}_{t}$ is assumed to be right-continuous.
} 


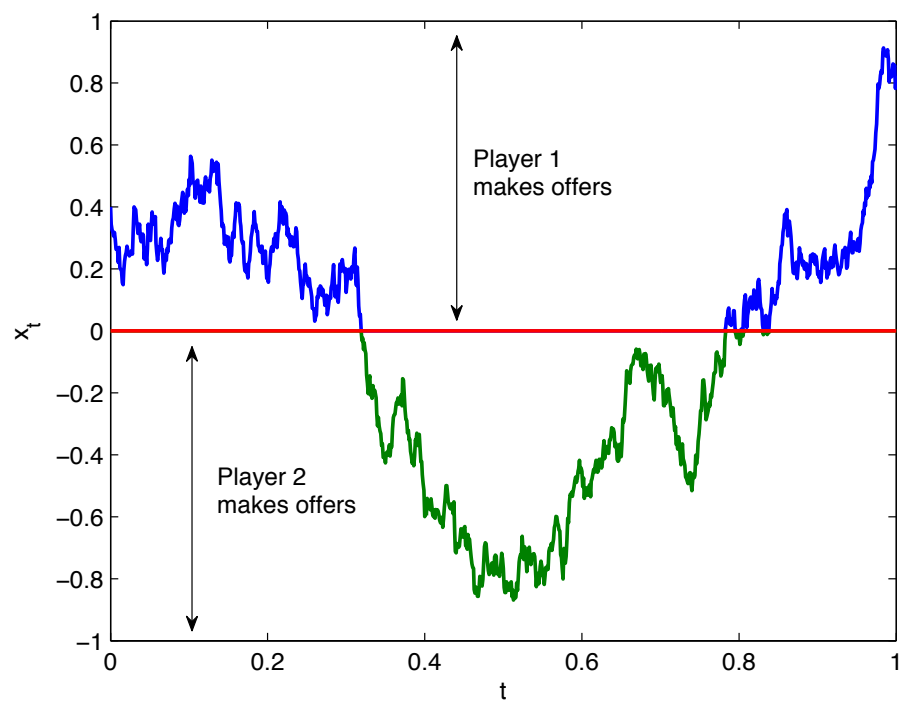

Figure 1: Sample path of $x_{t}$.

game ends and each player collects her payoff. If player 2 does not accept an offer between time 0 and $\tau_{1}$, then player 2 becomes the proposer from $\tau_{1}$ until $\tau_{2}:=\inf \left\{t>\tau_{1}: x_{t} \geq 0\right\}$. Bargaining continues this way, with players alternating in their right to make proposals until a player accepts an offer. Figure 1 plots a sample path of $x_{t}{ }^{5}$

Outcomes and equilibrium notion. An outcome of this bargaining game is a pair $(A, \eta)$, where $A \in \mathcal{A}:=\{A \subseteq[-1,1]: A$ is closed $\}$ is an agreement region and $\eta: A \rightarrow\left\{y \in \mathbb{R}_{+}^{2}:\right.$ $\left.y_{1}+y_{2}=1\right\}$ is a function mapping the agreement region to the set of possible divisions of the surplus. ${ }^{6}$ The agreement region $A$ determines the set of values of the state variable $x_{t}$ at which players reach an agreement: under outcome $(A, \eta)$ players reach an agreement at time $\tau(A):=\inf \left\{t \geq 0: x_{t} \in A\right\}$. The function $\eta(\cdot)=\left(\eta_{1}(\cdot), \eta_{2}(\cdot)\right)$ gives the share of the surplus that each player gets when they reach an agreement. The payoff that player $i$ gets

\footnotetext{
${ }^{5} \mathrm{~A}$ previous version of the paper showed that the main results in this paper characterizing equilibrium outcomes (Theorems 1 and 2) extend to settings in which $x_{t}$ follows a diffusion process of the form $d x_{t}=$ $\mu\left(x_{t}\right) d t+\sigma\left(x_{t}\right) d B_{t}$.

${ }^{6}$ The restriction that the agreement region $A$ must belong to the set $\mathcal{A}$ is only to get rid of trivial multiplicities - see footnote 9 in the Appendix.
} 
from outcome $(A, \eta)$ when the current relative bargaining power is $x$ is

$$
V_{i}(x)=\mathbb{E}\left[e^{-r \tau(A)} \eta_{i}\left(x_{\tau(A)}\right) \mid x_{0}=x\right]
$$

In what follows, I will denote an outcome $(A, \eta)$ as a triplet $\left(A, V_{1}, V_{2}\right)$, with $A \in \mathcal{A}$ and with $V_{i}$ satisfying (1) for $i=1,2$.

Let $T_{1}$ be the set of stopping times $\tau$ such that $x_{\tau} \in[0,1]$. Similarly, let $T_{2}$ be the set of stopping times $\tau$ such that $x_{\tau} \in[-1,0]$.

Definition 1 An outcome $\left(A, V_{1}, V_{2}\right)$ is an equilibrium if, for $i, j=1,2, j \neq i$,

$$
V_{i}(x)=\sup _{\tau \in T_{i}} \mathbb{E}\left[e^{-r \tau}\left(1-V_{j}\left(x_{\tau}\right)\right) \mid x_{0}=x\right] \forall x \in[-1,1] .
$$

In words, outcome $\left(A, V_{1}, V_{2}\right)$ is an equilibrium if, for $i=1,2$ and for all $x \in[-1,1], V_{i}(x)$ is equal to the value of the optimal stopping problem $\sup _{\tau \in T_{i}} \mathbb{E}\left[e^{-r \tau}\left(1-V_{j}\left(x_{\tau}\right)\right) \mid x_{0}=x\right]$.

The idea behind definition 1 is that the responder should be willing to accept proposals that give her what she expects to get by waiting until she regains the right to make offers. In other words, the responder's acceptance threshold must be equal to her expected continuation value. Since a proposer never offers the responder more than her acceptance threshold, the responder's payoff should always be equal to her expected continuation value. Definition 1 implies that this will occur under any equilibrium outcome. Indeed, suppose $x_{0}=x$ is such that player $i$ is the responder at $t=0$ and let $\tau(0):=\inf \left\{t \geq 0: x_{t}=0\right\}$. Note that $\tau(0) \leq \tau$ for all $\tau \in T_{i}$ when $x_{0}=x$. Equation (2) then implies that

$$
V_{i}(x)=\mathbb{E}\left[e^{-r \tau(0)} V_{i}(0) \mid x_{0}=x\right]
$$

regardless of whether players reach an agreement when $x_{t}=x$ or not (i.e., regardless of whether $x$ belongs to the agreement region $A$ or not).

On the other hand, when player $i$ is proposer she takes player $j$ 's acceptance threshold 
$V_{j}(x)$ as given. At each moment in time before she loses the right to make proposals, player $i$ has to decide whether to make an acceptable offer of $V_{j}\left(x_{t}\right)$ to her opponent and end the bargaining (keeping $1-V_{j}\left(x_{t}\right)$ for herself), or to delay the agreement until she can strike a better deal. Definition 1 says that an outcome $\left(A, V_{1}, V_{2}\right)$ is an equilibrium if the proposer always finds it optimal to delay when $x_{t} \notin A$, and always finds it optimal to make an acceptable offer when $x_{t} \in A$.

On bargaining games played in continuous time. It is well known that there are technical issues when modeling games in continuous time (e.g., Simon and Stinchcombe (1989)). One of these difficulties is that subgame perfection has less bite in continuoustime bilateral bargaining games than in their discrete-time counterparts. In discrete-time bargaining games à la Rubinstein (1982) the responder incurs a fixed cost of delay if she rejects the current offer, since she must wait one period to reach an agreement. This fixed cost of delay imposes restrictions on the strategies that players use in a subgame perfect equilibrium (SPE). In particular, in any SPE the responder will always accept offers that leave her indifferent between accepting or waiting until she becomes proposer.

In contrast, the responder doesn't face a fixed cost of delay when the game is in continuous time, since she can always accept a new offer within an arbitrarily short time period. As a result, in continuous time there are equilibria in which the responder doesn't accept offers equal to her expected continuation payoff of waiting until she becomes proposer. For instance, in continuous time the outcome in which player 1 always gets the entire surplus, regardless of the level of bargaining power, can be supported as an equilibrium. In this equilibrium player 1 only accepts offers that give her the entire surplus when she is responder, and only makes such offers when she is proposer. Against this strategy of player 1, player 2 can do no better than to always offer her opponent the entire surplus when she is proposer, and to accept offers that give her zero payoff when she is responder.

The ideas in the previous paragraph are not new. For instance, Bergin and MacLeod 
(1993) noted that in a continuous-time bargaining game with no restrictions on the timing of offers, any division of the surplus can be supported as an equilibrium outcome. Indeed, the division $(z, 1-z)$ with $z \in[0,1]$ can be supported by the strategy in which each player offers $z$ for player 1 and $1-z$ for player 2 at every time $t \geq 0$.

Beyond these complications, it is well-known that there are other difficulties analyzing games with observable actions in continuous time. As noted in the introduction, previous papers (e.g. Simon and Stinchcombe (1989), Bergin and MacLeod (1993)) deal with these difficulties by introducing frictions in the timing of moves (i.e., discrete grids, or inertia strategies). In contrast, in this paper I deal with these complexities by analyzing bargaining outcomes directly, and by building into the definition of equilibrium the restriction that the responder always accepts offers equal to her expected continuation value of waiting until she becomes proposer, just as in a SPE of a discrete-time bargaining game.

\section{Analysis}

\subsection{Equilibrium}

This section characterizes the equilibria of the model in Section 2. As a first step, the following result establishes that every equilibrium outcome must involve immediate agreement. All proofs are in the Appendix.

Proposition 1 (Immediate Agreement) Let $\left(A, V_{1}, V_{2}\right)$ be an equilibrium outcome. Then, it must be that $A=[-1,1]$.

Define $A_{1}^{*}:=[0,1], A_{2}^{*}:=[-1,0]$ and $A^{*}:=[-1,1]$. Note that $A_{i}^{*}$ is the set of values of $x$ at which player $i$ has proposal power. Recall that $\tau(0)=\inf \left\{t \geq 0: x_{t}=0\right\}$. 
Corollary 1 Let $\left(A, V_{1}, V_{2}\right)$ be an equilibrium outcome. Then, for $i, j=1,2, i \neq j$,

$$
V_{i}(x)= \begin{cases}1-V_{j}(x) & \text { if } x \in A_{i}^{*}, \\ \mathbb{E}\left[e^{-r \tau(0)}\left(1-V_{j}(0)\right) \mid x_{0}=x\right] & \text { if } x \notin A_{i}^{*} .\end{cases}
$$

Corollary 1 provides a partial characterization of the payoffs that can arise in an equilibrium of this model: player $i$ 's payoff when she is responder is given by the expected discounted value of waiting until $x_{t}$ reaches 0 and getting $1-V_{j}(0)$ of the surplus at that point. On the other hand, when player $i$ is making proposals she immediately makes an acceptable offer to her opponent, thus receiving a payoff of $1-V_{j}(x)$.

The next result builds on Proposition 1 and Corollary 1 and shows that there is a unique equilibrium.

Theorem 1 There is a unique equilibrium outcome $\left(A^{*}, V_{1}^{*}, V_{2}^{*}\right)$. For $i=1,2, V_{i}^{*}(\cdot)$ solves

$$
r V_{i}^{*}(x)= \begin{cases}\mu\left(V_{i}^{*}\right)^{\prime}(x)+\frac{1}{2} \sigma^{2}\left(V_{i}^{*}\right)^{\prime \prime}(x) & \text { if } x \notin A_{i}^{*}, \\ r+\mu\left(V_{i}^{*}\right)^{\prime}(x)+\frac{1}{2} \sigma^{2}\left(V_{i}^{*}\right)^{\prime \prime}(x) & \text { if } x \in A_{i}^{*},\end{cases}
$$

with $\left(V_{i}^{*}\right)^{\prime}(-1)=\left(V_{i}^{*}\right)^{\prime}(1)=0, \lim _{x \uparrow 0} V_{i}^{*}(x)=\lim _{x \downarrow 0} V_{i}^{*}(x)$ and $\lim _{x \uparrow 0}\left(V_{i}^{*}\right)^{\prime}(x)=\lim _{x \downarrow 0}\left(V_{i}^{*}\right)^{\prime}(x)$.

Equation (4) has the following interpretation. The left-hand side is player $i$ 's payoff measured in flow terms. When player $i$ is responder, her flow payoff is equal to the expected change in her continuation value, which is $\mu\left(V_{i}^{*}\right)^{\prime}(x)+\frac{1}{2} \sigma^{2}\left(V_{i}^{*}\right)^{\prime \prime}(x)$. When player $i$ is proposer, her flow payoff is equal to the flow rent $r$ she extracts from her ability to make offers plus the expected change in her continuation value. The boundary conditions at 0 imply that $V_{i}^{*}$ is continuous and differentiable on $[-1,1]$. The boundary conditions at -1 and 1 are a consequence of the process $x_{t}$ : since $x_{t}$ has reflecting boundaries, party $i$ 's payoff becomes flat as $x$ approaches either -1 or 1 . 
Equation (4) has a unique solution satisfying the boundary conditions in Theorem 1, given by

$$
V_{i}^{*}(x)= \begin{cases}a_{i} e^{-\alpha x}+b_{i} e^{\beta x} & \text { if } x \notin A_{i}^{*}, \\ 1+c_{i} e^{-\alpha x}+d_{i} e^{\beta x} & \text { if } x \in A_{i}^{*},\end{cases}
$$

where $\alpha=\left(\mu+\sqrt{\mu^{2}+2 r \sigma^{2}}\right) / \sigma^{2}, \beta=\left(-\mu+\sqrt{\mu^{2}+2 r \sigma^{2}}\right) / \sigma^{2}$, and where $\left(a_{i}, b_{i}, c_{i}, d_{i}\right)$ are constants determined by the four boundary conditions; see equation (A.6) in the Appendix for the full expression of $V_{1}^{*}(x)$.

The last result of this section presents comparative statics of $V_{i}^{*}$ with respect to the volatility of bargaining power. Recall that $A_{1}^{*}=[0,1]$ and $A_{2}^{*}=[-1,0]$.

Definition 2 The bargaining environment is favorable for player 1 (for player 2) if $\mu \geq 0$ (if $\mu \leq 0$ ).

Proposition 2 Suppose the bargaining environment is favorable for player 2 (for player 1). Then, there exists $x^{*} \geq 0\left(x^{*} \leq 0\right)$ such that $V_{1}^{*}(x)\left(V_{2}^{*}(x)\right)$ is increasing in $\sigma$ for all $x<x^{*}$ (for all $\left.x>x^{*}\right)$ and decreasing in $\sigma$ for all $x>x^{*}$ (for all $\left.x<x^{*}\right)$.

The intuition behind Proposition 2 is as follows. When the bargaining environment is favorable to the proposer, an increase in volatility raises the chances that the responder will recover bargaining power. This improves the responder's bargaining position, and allows her to obtain a better deal in the negotiations. Figure 2 illustrates for the special case with $\mu=0$.

The next result provides comparative statics with respect to the drift $\mu$ of $x_{t}$.

Proposition 3 For all $x \in[-1,1], V_{1}^{*}(x)$ is increasing in $\mu$ and $V_{2}^{*}(x)$ is decreasing in $\mu$. 


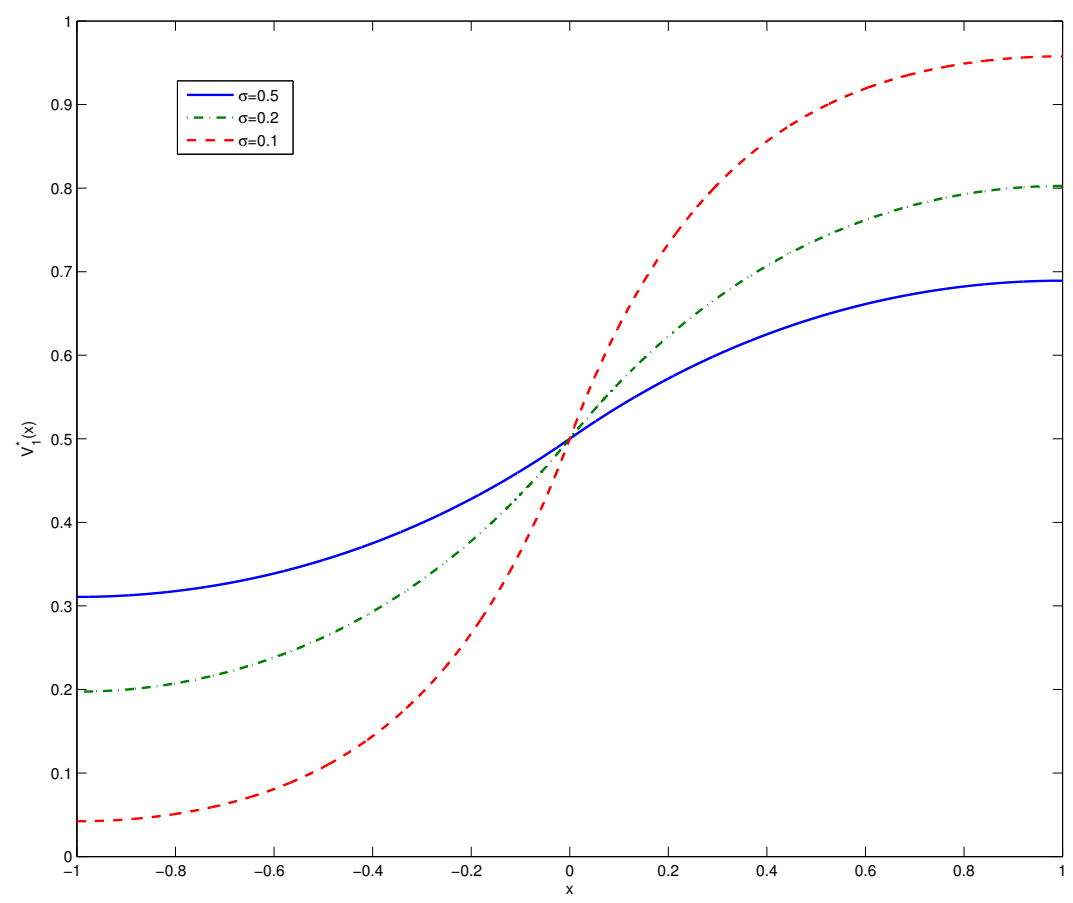

Figure 2: Payoffs $V_{1}^{*}$. Parameters: $\mu=0$ and $r=0.05$.

\subsection{Political bargaining with supermajority rules}

This section uses the model above to study political bargaining with supermajority rules. Two political parties, $i=1,2$, have to bargain over which policy in $[0,1]$ to implement. For $i=1,2$, party $i$ 's preference over policies $z \in[0,1]$ are represented by $u_{i}(z)$, with $u_{1}(z)=1-z$ and $u_{2}(z)=z$. Parties are expected utility maximizers and discount future payoffs at the common rate $r>0$.

The bargaining protocol is the same as above. Process $x_{t}$ evolves as Brownian motion with drift $\mu$, volatility $\sigma>0$ and with reflecting boundaries at -1 and 1 . At each time $t \geq 0$, party 1 has proposal power if $x_{t} \geq 0$ and party 2 has proposal power if $x_{t}<0$. In this political bargaining setting, I interpret the process $x_{t}$ as measuring the fraction of legislators supporting each party at any moment in time: party 1 has the support of a majority of 
legislators when $x_{t}>0$, while party 2 has the support of a majority when $x_{t}<0$. The assumption that the parties' legislative support is time-varying approximates a setting in which the parties' popularity is changing over time, and in which these changes in popularity influence the party that each individual legislator chooses to support. ${ }^{7}$

Supermajority rules. A supermajority rule is a number $\phi>0$ such that party 1 can unilaterally implement a policy at time $t$ if $x_{t} \geq \phi$ and party 2 can unilaterally implement a policy at time $t$ if $x_{t} \leq-\phi$. Under a supermajority rule a party with enough support can implement policies unilaterally, without the approval of its opponent. On the other hand, parties need to bargain over which policy to implement when neither of them has enough support (i.e., when $\left.x_{t} \in(-\phi, \phi)\right)$.

Theorem A1 in Appendix A.2 shows that this model has a unique equilibrium outcome, and characterizes the parties' equilibrium payoffs. Here, I focus on how supermajority rules affect the parties' equilibrium payoffs. To emphasize the dependence on the supermajority requirement, in what follows I write $V_{i}^{\phi}(\cdot)$ to denote party $i$ 's equilibrium payoff under supermajority rule $\phi$.

Proposition 4 Fix two supermajority rules $\phi<1$ and $\phi^{\prime} \in(\phi, 1]$. Then, there exists $\tilde{x} \in$ $(-\phi, \phi)$ such that $V_{1}^{\phi^{\prime}}(x)>V_{1}^{\phi}(x)$ for all $x \in(-\phi, \tilde{x})$ and $V_{2}^{\phi^{\prime}}(x)>V_{2}^{\phi}(x)$ for all $x \in\left(\tilde{x}, \phi^{\prime}\right)$.

Proposition 4 shows that more stringent supermajority rules benefit weaker parties (and hurt stronger parties): a party whose political power is low enough always prefers a supermajority rule that is harder to reach. Intuitively, an increase in $\phi$ makes it is less likely that the stronger party will obtain the required supermajority.

\footnotetext{
${ }^{7}$ In Ortner (2017b), I use a similar model to study how upcoming elections affect the outcomes of political negotiations.
} 


\section{Discrete-time game}

In this section I study the discrete-time version of the continuous-time model in Section $2 .{ }^{8}$ The goal is to show that the equilibrium of the continuous-time formulation corresponds to the limiting subgame perfect equilibrium of the discrete-time bargaining game, when players can make offers arbitrarily frequently. Hence, the tractable equilibrium of the continuoustime game provides a good approximation of settings with small bargaining frictions.

The discrete-time game is as follows. Two players, $i=1,2$, bargain over how to divide a perfectly divisible surplus of size 1 . Time is continuous but players can only make offers at points on the grid $T(\Delta)=\{0, \Delta, 2 \Delta, \ldots\}$, where $\Delta>0$ measures the time between bargaining rounds. As in Sections 2 and 3.1, $x_{t}$ evolves as a Brownian motion with drift $\mu$ and volatility $\sigma>0$, with reflecting boundaries at -1 and 1 .

The bargaining protocol is as follows. At any time $t \in T(\Delta)$ the realization of $x_{t}$ determines the identity of the proposer: player 1 is proposer at time $t$ if $x_{t} \geq 0$, and player 2 is proposer at time $t$ if $x_{t}<0$. The proposer makes an offer $z \in\left\{y \in \mathbb{R}_{+}^{2}: y_{1}+y_{2}=1\right\}$. The responder can either accept or reject the offer. If the responder accepts the offer, the game ends and players collect their payoffs. If the responder rejects the offer, the game moves to period $t+\Delta$. Players are risk neutral expected utility maximizers and share the same discount factor $e^{-r \Delta}$ (with $r>0$ ).

Let $\Gamma_{\Delta}$ denote the bargaining game with time interval $\Delta>0$. Recall that $A_{1}^{*}=[0,1]$ and $A_{2}^{*}=[-1,0]$. The following result characterizes the unique SPE of $\Gamma_{\Delta}$.

Theorem 2 For any $\Delta>0, \Gamma_{\Delta}$ has a unique SPE. In the unique SPE players reach an immediate agreement.

For $i=1,2$, let $V_{i}^{\Delta}(x)$ denote player $i$ 's SPE payoff at a subgame in which players have not yet reached an agreement and in which current bargaining power is $x$. Then, for

\footnotetext{
${ }^{8}$ For brevity, I don't analyze the discrete-time version of the model in Section 3.2.
} 
$i, j=1,2, j \neq i$

$$
V_{i}^{\Delta}(x)= \begin{cases}e^{-r \Delta} \mathbb{E}\left[V_{i}^{\Delta}\left(x_{t+\Delta}\right) \mid x_{t}=x\right] & \text { if } x \notin A_{i}^{*}, \\ 1-e^{-r \Delta} \mathbb{E}\left[V_{j}^{\Delta}\left(x_{t+\Delta}\right) \mid x_{t}=x\right] & \text { if } x \in A_{i}^{*},\end{cases}
$$

The content of Theorem 2 can be described as follows. In a subgame perfect equilibrium the responder only accepts offers that give her a payoff at least as large as her expected continuation value of waiting one period. Knowing this, the proposer always makes the lowest offer that the responder is willing to accept, and the game ends with an immediate agreement.

The last result shows that the unique SPE of $\Gamma_{\Delta}$ converges to the equilibrium in Theorem 1 as $\Delta \rightarrow 0$.

Theorem 3 For $i=1,2, V_{i}^{\Delta}$ converges uniformly to $V_{i}^{*}$ as $\Delta \rightarrow 0$.

\section{A Appendix}

\section{A.1 Proofs of Section 3.1}

Proof of Proposition 1. Suppose $\left(A, V_{1}, V_{2}\right)$ is an equilibrium and assume by contradiction that $A$ is a strict subset of $[-1,1]$. Since $[-1,1] \backslash A$ is open (because $A \in \mathcal{A}=\{A \subseteq[-1,1]: A$ is closed $\})$, there exists an open interval $(\underline{y}, \bar{y}) \subset[-1,1]$ such that $(\underline{y}, \bar{y}) \nsubseteq A$, so $\tau(A)>0$ whenever $x_{0} \in(\underline{y}, \bar{y}) .{ }^{9}$ Define $W(x):=V_{1}(x)+V_{2}(x)=\mathbb{E}\left[e^{-r \tau(A)} \mid x_{0}=x\right]$, and note that $W(x)<1$ for all $x \in(\underline{y}, \bar{y})$. Thus, $V_{1}(x)+V_{2}(x)<1$ for all $x \in(\underline{y}, \bar{y})$. But this implies that, when $x_{t} \in(\underline{y}, \bar{y})$, proposer $i$ is better off offering $V_{j}\left(x_{t}\right)$ to her opponent (and obtaining a payoff of $1-V_{j}\left(x_{t}\right)>V_{i}\left(x_{t}\right)$ for herself $)$ than delaying. Therefore, $\left(A, V_{1}, V_{2}\right)$ cannot be an equilibrium outcome.

\footnotetext{
${ }^{9}$ If I allow for agreement regions $A$ that don't belong to the set $\mathcal{A}$, then there would be equilibrium agreement regions of the form $\mathbb{R} \backslash Z$, where $Z$ is a set of measure zero. The restriction to $A \in \mathcal{A}$ rules out this (trivial) source of multiplicity.
} 
Proof of Corollary 1. Let $\left(A^{*}, V_{1}, V_{2}\right)$ be an equilibrium outcome. Equation (3) then implies that, for all $x \notin A_{i}^{*}$,

$$
V_{i}(x)=\mathbb{E}\left[e^{-r \tau(0)} V_{i}(0) \mid x_{0}=x\right]=\mathbb{E}\left[e^{-r \tau(0)}\left(1-V_{j}(0)\right) \mid x_{0}=x\right],
$$

where I used $V_{1}(x)+V_{2}(x)=1$ for all $x$ (which follows from Proposition 1). Finally, Proposition 1 also implies that $V_{i}(x)=1-V_{j}(x)$ for all $x \in A_{i}^{*}$.

Before moving to the proof of Theorem 1, I present two preliminary lemmas. For any $y, z \in(-1,1)$, let $\tau_{y}:=\inf \left\{t: x_{t}=y\right\}$ and $\tau_{y z}=\inf \left\{t: x_{t}=y\right.$ or $\left.x_{t}=z\right\}$.

Lemma A1 Let $g: \mathbb{R} \rightarrow \mathbb{R}$ be a bounded function, and let $f(\cdot)$ be the solution to

$$
r f(x)=\mu f^{\prime}(x)+\frac{\sigma^{2}}{2} f^{\prime \prime}(x) .
$$

(i) If $f^{\prime}(-1)=0$ and $f(y)=g(y)$, then for all $x \in[-1, y], f(x)=\mathbb{E}\left[e^{-r \tau_{y}} g(y) \mid x_{0}=x\right]$.

(ii) If $f^{\prime}(1)=0$ and $f(y)=g(y)$, then for all $x \in[y, 1], f(x)=\mathbb{E}\left[e^{-r \tau_{y}} g(y) \mid x_{0}=x\right]$.

(iii) If $f(x)=g(x)$ for $x=y, z$ with $0 \leq y<z \leq 1$, then for all $x \in[y, z], f(x)=$ $\mathbb{E}\left[e^{-r \tau_{y z}} g(y) \mid x_{0}=x\right]$.

Proof. I prove part (i) of the Lemma. The proofs of parts (ii) and (iii) are symmetric and omitted. Suppose $f(\cdot)$ solves the differential equation and boundary conditions in the statement of the Lemma. By Ito's Lemma, for all $x_{t} \in[-1, y]$,

$$
\begin{aligned}
d\left(e^{-r t} f\left(x_{t}\right)\right) & =e^{-r t}\left(-r f\left(x_{t}\right)+\mu f^{\prime}\left(x_{t}\right)+\frac{\sigma^{2}}{2} f^{\prime \prime}\left(x_{t}\right)\right) d t+f^{\prime}(-1) d L_{t}+e^{-r t} f^{\prime}\left(x_{t}\right) \sigma d B_{t} \\
& =e^{-r t} f^{\prime}\left(x_{t}\right) \sigma d B_{t}
\end{aligned}
$$

where $L_{t}$ is the "regulator process" that controls reflection at $x=-1$ (see Harrison (1985) 
for details). Since $e^{-r t} f\left(x_{t}\right)=f\left(x_{0}\right)+\int_{0}^{t} d\left(e^{-r t} f\left(x_{t}\right)\right)$, it follows that

$$
e^{-r \tau_{y}} f\left(x_{\tau_{y}}\right)=f\left(x_{0}\right)+\int_{0}^{\tau_{y}} e^{-r s} f^{\prime}\left(x_{s}\right) \sigma d B_{s}
$$

Note that $\int_{0}^{t} e^{-r s} f^{\prime}\left(x_{s}\right) \sigma d B_{s}$ is a Martingale with expectation zero. Since $\mathbb{E}\left[\tau_{y}\right]<\infty$ and since $f \in C^{2}$ and has bounded support, it follows from the Optional Sampling Theorem (Kallenberg (2006), Theorem 7.29) that $\mathbb{E}\left[\int_{0}^{\tau_{y}} e^{-r s} f^{\prime}\left(x_{s}\right) \sigma d B_{s}\right]=0$. The result then follows from taking expectations in both sides of (A.2) and noting that $f\left(x_{\tau_{y}}\right)=f(y)=g(y)$.

An implication of Lemma A1 is that the function $f(x)=\mathbb{E}\left[e^{-r \tau_{y}} g(y) \mid x_{0}=x\right]$ solves (A.1) for all $x \in[-1, y]$ and with boundary conditions $f^{\prime}(-1)=0$ and $f(y)=g(y)$, and solves (A.1) for all $x \in[y, 1]$ and with boundary conditions $f^{\prime}(1)=0$ and $f(y)=g(y) .{ }^{10}$

The following Lemma, which I use in the proofs of Theorem 1 and of Proposition 2, studies properties of solutions to the ODE in equation (A.1).

Lemma A2 Let $U$ be a solution to (A.1) with parameters $(\hat{\mu}, \hat{\sigma})$ and with $U^{\prime \prime}(x)>0$ for all $x$, and let $W$ be a solution to (A.1) with parameters $(\tilde{\mu}, \tilde{\sigma})$, with $\tilde{\sigma} \geq \hat{\sigma}$, and with $W^{\prime \prime}(x)>0$ for all $x$.

(i) Suppose that $\tilde{\mu} \geq \hat{\mu}$, and that $U^{\prime} \geq 0$ and $W^{\prime} \geq 0$. If $U(y) \geq W(y)$ and $U^{\prime}(y) \geq W^{\prime}(y)$ for some $y$, with at least one inequality strict if $(\widetilde{\mu}, \widetilde{\sigma})=(\hat{\mu}, \hat{\sigma})$, then $U^{\prime}(x)>W^{\prime}(x)$ for all $x>y$, and so $U(x)>W(x)$ for all $x>y$.

(ii) Suppose that $\tilde{\mu} \leq \hat{\mu}$, and that $U^{\prime} \leq 0$ and $W^{\prime} \leq 0$. If $U(y) \geq W(y)$ and $U^{\prime}(y) \leq W^{\prime}(y)$ for some $y$, with at least one inequality strict if $(\widetilde{\mu}, \widetilde{\sigma})=(\hat{\mu}, \hat{\sigma})$, then $U^{\prime}(x)<W^{\prime}(x)$ for all $x<y$, and so $U(x)>W(x)$ for all $x<y$.

Proof. I prove part (i) of the lemma. The proof of part (ii) is symmetric and omitted. To prove part (i), I start by showing that there exists $\eta>0$ such that $U^{\prime}(x)>W^{\prime}(x)$ for all

\footnotetext{
${ }^{10}$ This follows because differential equation (A.1) with boundary conditions in part (i) or part (ii) of Lemma A1 has a unique solution.
} 
$x \in(y, y+\eta)$. Since $U, W$ are continuously differentiable, this is true when $U^{\prime}(y)>W^{\prime}(y)$. Suppose that $U^{\prime}(y)=W^{\prime}(y)$. Since $U$ and $W$ solve (A.1) (with parameters $(\hat{\mu}, \hat{\sigma})$ and $(\widetilde{\mu}, \widetilde{\sigma})$, respectively),

$$
W^{\prime \prime}(y)=\frac{2\left(r W(y)-\widetilde{\mu} W^{\prime}(y)\right)}{\widetilde{\sigma}^{2}}<\frac{2\left(r U(y)-\hat{\mu} U^{\prime}(y)\right)}{\hat{\sigma}^{2}}=U^{\prime \prime}(y),
$$

where the strict inequality follows since $(\widetilde{\mu}, \widetilde{\sigma}) \geq(\hat{\mu}, \hat{\sigma}), U(y) \geq W(y)$ (with at least one inequality strict), $U^{\prime \prime}, W^{\prime \prime}>0$, and $U^{\prime}, W^{\prime} \geq 0$. Since $U^{\prime}(y)=W^{\prime}(y)$ and $U^{\prime \prime}(y)>W^{\prime \prime}(y)$, there exists $\eta>0$ such that $U^{\prime}(x)>W^{\prime}(x) \forall x \in(y, y+\eta)$.

Suppose next that part (i) in the lemma is not true and let $y_{1}$ be the smallest point strictly above $y$ with $U^{\prime}\left(y_{1}\right)=W^{\prime}\left(y_{1}\right)$. By the paragraph above, $y_{1} \geq y+\eta>y$. It follows that $U^{\prime}(x)>W^{\prime}(x)$ for all $x \in\left(y, y_{1}\right)$, so $U\left(y_{1}\right)>W\left(y_{1}\right)$. Note then that

$$
W^{\prime \prime}\left(y_{1}\right)=\frac{2\left(r W\left(y_{1}\right)-\widetilde{\mu} W^{\prime}\left(y_{1}\right)\right)}{\widetilde{\sigma}^{2}}<\frac{2\left(r U\left(y_{1}\right)-\hat{\mu} U^{\prime}\left(y_{1}\right)\right)}{\hat{\sigma}^{2}}=U^{\prime \prime}\left(y_{1}\right),
$$

where the inequality follows since $U\left(y_{1}\right)>W\left(y_{1}\right),(\widetilde{\mu}, \widetilde{\sigma}) \geq(\hat{\mu}, \hat{\sigma}), U^{\prime \prime}, W^{\prime \prime}>0$ and $U^{\prime}, W^{\prime} \geq$ 0 . Since $U^{\prime}\left(y_{1}\right)=W^{\prime}\left(y_{1}\right)$ and $U^{\prime \prime}\left(y_{1}\right)>W^{\prime \prime}\left(y_{1}\right)$, it must be that $U^{\prime}\left(y_{1}-\varepsilon\right)<W^{\prime}\left(y_{1}-\varepsilon\right)$ for $\varepsilon>0$ small, a contradiction. Thus, $U^{\prime}(x)>W^{\prime}(x) \forall x>y$.

Proof of Theorem 1. Let $\left(A^{*}, V_{1}, V_{2}\right)$ be an equilibrium outcome. By Corollary 1 , for $i=1,2$ and for all $x \notin A_{i}^{*}$ it must be that $V_{i}(x)=\mathbb{E}\left[e^{-r \tau(0)}\left(1-V_{j}(0)\right) \mid x_{0}=x\right]$. By Lemma A1, for all $x \notin A_{i}^{*}, V_{i}(x)$ solves

$$
r V_{i}(x)=\mu V_{i}^{\prime}(x)+\frac{1}{2} \sigma^{2} V_{i}^{\prime \prime}(x)
$$

with boundary conditions $V_{i}(0)=1-V_{j}(0)$ and $V_{1}^{\prime}(-1)=V_{2}^{\prime}(1)=0$. Since $V_{i}(x)=1-V_{j}(x)$ 
for all $x$, this implies that, for $i=1,2$

$$
r V_{i}(x)= \begin{cases}\mu V_{i}^{\prime}(x)+\frac{1}{2} \sigma^{2} V_{i}^{\prime \prime}(x) & \text { if } x \notin A_{i}^{*}, \\ r+\mu V_{i}^{\prime}(x)+\frac{1}{2} \sigma^{2} V_{i}^{\prime \prime}(x) & \text { if } x \in A_{i}^{*},\end{cases}
$$

with $V_{i}^{\prime}(-1)=V_{i}^{\prime}(1)=0$ and $\lim _{x \uparrow 0} V_{i}(x)=\lim _{x \downarrow 0} V_{i}(x)$. The general solution to (A.4) is

$$
V_{i}(x)= \begin{cases}C_{i} e^{-\alpha x}+D_{i} e^{\beta x} & \text { if } x \notin A_{i}^{*}, \\ 1+E_{i} e^{-\alpha x}+F_{i} e^{\beta x} & \text { if } x \in A_{i}^{*},\end{cases}
$$

where $\alpha=\left(\mu+\sqrt{\mu^{2}+2 r \sigma^{2}}\right) / \sigma^{2}>0$ and $\beta=\left(-\mu+\sqrt{\mu^{2}+2 r \sigma^{2}}\right) / \sigma^{2}>0$, and where $\left(C_{i}, D_{i}, E_{i}, F_{i}\right)$ are constants. For $i=1,2$, let $V_{i}^{*}$ be the unique solution to (A.4) satisfying the boundary conditions in the statement of Theorem 1. It can be checked that

$$
V_{1}^{*}(x)= \begin{cases}\frac{\beta e^{-\alpha x}+e^{\alpha+\beta} \alpha e^{\beta x}}{(\alpha+\beta)\left(1+e^{\alpha+\beta}\right)} & \text { if } x \in[-1,0), \\ 1-\frac{e^{\alpha+\beta} \beta e^{-\alpha x}+\alpha e^{\beta x}}{(\alpha+\beta)\left(1+e^{\alpha+\beta}\right)} & \text { if } x \in[0,1],\end{cases}
$$

and $V_{2}^{*}(x)=1-V_{1}^{*}(x)$. Note that $\left(V_{1}^{*}\right)^{\prime \prime}(x)>0$ for all $x \in[-1,0)$, and that $\left(V_{2}^{*}\right)^{\prime \prime}(x)>0$ for all $x \in(0,1]$ (these inequalities follow since $\alpha>0$ and $\beta>0$ ).

To establish that $\left(A^{*}, V_{1}^{*}, V_{2}^{*}\right)$ is an equilibrium, I show that, for all $x \in A_{1}^{*}, V_{1}^{*}(x)$ solves

$$
V_{1}^{*}(x)=\sup _{\tau \in T_{1}} \mathbb{E}\left[e^{-r \tau}\left(1-V_{2}^{*}\left(x_{\tau}\right)\right) \mid x_{0}=x\right]
$$

The proof that $V_{2}^{*}$ also solves the optimal stopping problem is symmetric and omitted. To see that $V_{1}^{*}$ solves (A.7), let $G_{2}(x)$ be a solution to the ODE in equation (A.1), with boundary conditions $G_{2}(1)=V_{2}^{*}(1)$ and $G_{2}^{\prime}(1)=\left(V_{2}^{*}\right)^{\prime}(1)=0$. The solution to this differential equation is

$$
G_{2}(x)=\frac{e^{\alpha+\beta} \beta e^{-\alpha x}+\alpha e^{\beta x}}{(\alpha+\beta)\left(1+e^{\alpha+\beta}\right)}
$$


Note that, for all $x \in[0,1], G_{2}(x)=V_{2}^{*}(x)$. Consider the optimal stopping problem

$$
G_{1}(x)=\sup _{\tau \in T} \mathbb{E}\left[e^{-r \tau}\left(1-G_{2}\left(x_{\tau}\right)\right) \mid x_{0}=x\right]
$$

where $T$ is the set of all stopping times. Since $G_{2}(x)=V_{2}^{*}(x)$ for all $x \in[0,1]$ and since $T_{1}$ is the set of stopping times $\tau$ such that $x_{\tau} \in[0,1], G_{1}(x) \geq \sup _{\tau \in T_{1}} \mathbb{E}\left[e^{-r \tau}\left(1-V_{2}^{*}\left(x_{\tau}\right)\right) \mid x_{0}=x\right]$. Therefore, in order to show that $V_{1}^{*}$ solves (A.7), it suffices to show that $V_{1}^{*}(x)=G_{1}(x)$.

The function $V_{1}^{*}$ is twice differentiable, with a continuous first derivative. Note that $V_{1}^{*}(x) \geq 1-G_{2}(x)$ for all $x \in[-1,1]$, with strict inequality for all $x \in[-1,0)$. Indeed, $V_{1}^{*}(x)=1-V_{2}^{*}(x)=1-G_{2}(x)$ for all $x \in[0,1]$. Moreover, note that $\left(V_{1}^{*}\right)^{\prime}(0)=-\left(V_{2}^{*}\right)^{\prime}(0)=$ $-G_{2}^{\prime}(0)$ (the first equality follows since $V_{1}^{*}(x)+V_{2}^{*}(x)=1 \forall x$ and the second follows since $\left.G_{2}(x)=V_{2}^{*}(x) \forall x \in[0,1]\right)$. Since $V_{1}^{*}(x)$ is convex for all $x \in[-1,0)$ and $1-G_{2}(x)$ is concave for all $x$ (see equations (A.6) and (A.8)), it follows that $-G_{2}^{\prime}(x)>\left(V_{1}^{*}\right)^{\prime}(x)$ for all $x<0$, and so $V_{1}^{*}(x)>1-G_{2}(x)$ for all $x<0$. Finally, note that $V_{1}^{*}(x)$ satisfies

$$
-r V_{1}^{*}(x)+\mu\left(V_{1}^{*}\right)^{\prime}(x)+\frac{1}{2} \sigma^{2}\left(V_{1}^{*}\right)^{\prime \prime} \leq 0, \text { with equality on } x \in[-1,0) \text {. }
$$

Therefore, by standard verification theorems (e.g., Theorem 3.17 in Shiryaev (2007)), $V_{1}^{*}$ is the value function of the optimal stopping problem (A.9). Hence, $V_{1}^{*}$ also solves (A.7).

Next, I show that $\left(A^{*}, V_{1}^{*}, V_{2}^{*}\right)$ is the unique equilibrium. By Proposition 1, any equilibrium outcome $\left(A, V_{1}, V_{2}\right)$ must have $A=A^{*}=[-1,1]$. Let $\left(A^{*}, V_{1}, V_{2}\right)$ be an equilibrium outcome different from $\left(A^{*}, V_{1}^{*}, V_{2}^{*}\right)$. The discussion at the beginning of the proof implies that, for $i=1,2, V_{i}$ solves (A.4), with $V_{i}^{\prime}(-1)=V_{i}^{\prime}(1)=0$ and $\lim _{x \uparrow 0} V_{i}(x)=\lim _{x \downarrow 0} V_{i}(x)$. Since $\left(V_{1}, V_{2}\right) \neq\left(V_{1}^{*}, V_{2}^{*}\right)$, it must be that $\lim _{x \uparrow 0} V_{i}^{\prime}(x) \neq \lim _{x \downarrow 0} V_{i}^{\prime}(x)$. Since $V_{1}(x)+V_{2}(x)=1 \forall x$, there are two cases to consider: (i) $\lim _{x \uparrow 0} V_{1}^{\prime}(x)<\lim _{x \downarrow 0} V_{1}^{\prime}(x)=\lim _{x \downarrow 0}-V_{2}^{\prime}(x)$, or (ii) $\lim _{x \uparrow 0} V_{1}^{\prime}(x)>\lim _{x \downarrow 0} V_{1}^{\prime}(x)=\lim _{x \downarrow 0}-V_{2}^{\prime}(x)$.

Consider case (i). For every $\varepsilon \in(0,1]$ let $\tau^{\varepsilon}:=\inf \left\{t \geq 0: x_{t} \geq \varepsilon\right\}$ and note that $\tau^{\varepsilon} \in T_{1}$. Let $V_{1}^{\varepsilon}(x):=\mathbb{E}\left[e^{-r \tau^{\varepsilon}}\left(1-V_{2}\left(x_{\tau^{\varepsilon}}\right)\right) \mid x_{0}=x\right]$. By Lemma A1, for all $x \leq \varepsilon, V_{1}^{\varepsilon}(x)$ solves the 
ODE in equation (A.1) with $\left(V_{1}^{\varepsilon}\right)^{\prime}(-1)=0$ and $V_{1}^{\varepsilon}(\varepsilon)=1-V_{2}(\varepsilon)=V_{1}(\varepsilon)$. One can check that, for all $x \leq \epsilon, V_{1}^{\varepsilon}(x)=\frac{V_{1}(\varepsilon)}{\beta e^{-\alpha \varepsilon}+\alpha e^{\alpha+\beta} e^{\beta \varepsilon}}\left(\beta e^{-\alpha x}+\alpha e^{\alpha+\beta} e^{\beta x}\right)$. Note that $\left(V_{1}^{\varepsilon}\right)^{\prime}(x)>0$ and $\left(V_{1}^{\varepsilon}\right)^{\prime \prime}(x)>0$.

Let $F_{1}(x)$ be the solution to the ODE in equation (A.1), with $F_{1}^{\prime}(-1)=V_{1}^{\prime}(-1)=0$ and $F_{1}(-1)=V_{1}(-1)$. Note that $F_{1}(x)=V_{1}(x)$ for all $x \leq 0$. Moreover, since $\lim _{x \uparrow 0} V_{1}^{\prime}(x)<$ $\lim _{x \downarrow 0}-V_{2}^{\prime}(x)$, there exists $\varepsilon>0$ small enough such that $F_{1}(\varepsilon)<V_{1}^{\varepsilon}(\varepsilon)=1-V_{2}(\varepsilon)$. I now use this to show that, for all $x<0$,

$$
V_{1}^{\varepsilon}(x)=\mathbb{E}\left[e^{-r \tau^{\varepsilon}}\left(1-V_{2}\left(x_{\tau^{\varepsilon}}\right)\right) \mid x_{0}=x\right]>\mathbb{E}\left[e^{-r \tau(0)}\left(1-V_{2}(0)\right) \mid x_{0}=x\right]=V_{1}(x)=F_{1}(x) .
$$

Note that the inequality in (A.10) implies that $V_{1}(x)$ is not a solution to the optimal stopping problem (2). Hence, if (A.10) holds, $\left(A^{*}, V_{1}, V_{2}\right)$ cannot be an equilibrium outcome.

To show that the inequality in (A.10) holds, I start by showing that $V_{1}^{\varepsilon}(-1)>F_{1}(-1)$. Note first that it cannot be that $V_{1}^{\varepsilon}(-1)=F_{1}(-1)$. Indeed, $F_{1}$ and $V_{1}^{\varepsilon}$ both solve the ODE in (A.1), with $F_{1}^{\prime}(-1)=0=\left(V_{1}^{\varepsilon}\right)^{\prime}(-1)$. If $V_{1}^{\varepsilon}(-1)=F_{1}(-1)$, then $V_{1}^{\epsilon}$ and $F_{1}$ would both be the solution to the same ODE, with the same boundary conditions. This would imply that $V_{1}^{\epsilon}(x)=F_{1}(x)$ for all $x \leq \varepsilon$, a contradiction to $F_{1}(\varepsilon)<V_{1}^{\varepsilon}(\varepsilon)$. Suppose next that $V_{1}^{\varepsilon}(-1)<F_{1}(-1)$. Since $F_{1}^{\prime}(-1)=0=\left(V_{1}^{\varepsilon}\right)^{\prime}(-1)$, Lemma A2(i) implies that $V_{1}^{\epsilon}(x)<F_{1}(x)$ for all $x \in(-1, \varepsilon]$, which contradicts $V_{1}^{\varepsilon}(\varepsilon)>F_{1}(\varepsilon)$. Hence, $V_{1}^{\varepsilon}(-1)>F_{1}(-1)$. Finally, since $V_{1}^{\varepsilon}(-1)>F_{1}(-1)$ and $\left(V_{1}^{\varepsilon}\right)^{\prime}(-1)=F_{1}^{\prime}(-1)=0$, Lemma A2(i) implies that $V_{1}^{\varepsilon}(x)>F_{1}(x)$ for all $x<0$.

In case (ii), a symmetric argument establishes that there exists $\xi>0$ such that $V_{2}^{\xi}(x)=$ $\mathbb{E}\left[e^{-r \tau^{\xi}}\left(1-V_{1}\left(x_{\tau^{\xi}}\right)\right) \mid x_{0}=x\right]>V_{2}(x)$ for all $x>0$, where $\tau^{\xi}:=\inf \left\{t \geq 0: x_{t} \leq-\xi\right\} .{ }^{11}$ Hence, in this case $\left(A^{*}, V_{1}, V_{2}\right)$ cannot be an equilibrium outcome either.

\footnotetext{
${ }^{11}$ The symmetric argument for this uses Lemma A2(ii) instead of Lemma A2(i).
} 
Proof of Proposition 2. Suppose $\mu \leq 0$. Using equation (A.6), it can be shown that

$$
\frac{\partial V_{1}^{*}(0)}{\partial \sigma}=-\mu \frac{\left(4 e^{\frac{2 \sqrt{\mu^{2}+2 r \sigma^{2}}}{\sigma^{2}}} \mu^{2} \sqrt{\mu^{2}+2 r \sigma^{2}}+r \sigma^{2}\left(\left(-1+e^{\frac{4 \sqrt{\mu^{2}+2 r \sigma^{2}}}{\sigma^{2}}}\right) \sigma^{2}+4 e^{\frac{2 \sqrt{\mu^{2}+2 r \sigma^{2}}}{\sigma^{2}}} \sqrt{\mu^{2}+2 r \sigma^{2}}\right)\right)}{\left(1+e^{\frac{2 \sqrt{\mu^{2}+2 r \sigma^{2}}}{\sigma^{2}}}\right)^{2} \sigma^{3}\left(\mu^{2}+2 r \sigma^{2}\right)^{3 / 2}} \geq 0
$$

where the inequality follows since $\mu \leq 0$. Fix $\hat{\sigma}<\widetilde{\sigma}$ and let $\hat{V}_{1}^{*}$ and $\widetilde{V}_{1}^{*}$ be player 1's payoff when the volatility of $x_{t}$ is $\hat{\sigma}$ and $\widetilde{\sigma}$, respectively. By the derivative above, $\widetilde{V}_{1}^{*}(0) \geq \hat{V}_{1}^{*}(0)$. By Theorem 1, $\hat{V}_{1}^{*}$ and $\widetilde{V}_{1}^{*}$ solve (A.1) on $[-1,0]$ (but with different values of volatility), with $\left(\widetilde{V}_{1}^{*}\right)^{\prime}(-1)=\left(\hat{V}_{1}^{*}\right)^{\prime}(-1)=0$. Moreover, by (A.6), $\hat{V}_{1}^{*}$ and $\widetilde{V}_{1}^{*}$ are strictly convex and increasing for all $x<0$.

I now show that $\widetilde{V}_{1}^{*}(x)>\hat{V}_{1}^{*}(x) \forall x[-1,0)$. Note first that it must be that $\widetilde{V}_{1}^{*}(-1)>$ $\hat{V}_{1}^{*}(-1)$ : if $\hat{V}_{1}^{*}(-1) \geq \widetilde{V}_{1}^{*}(-1)$, then Lemma A2 (i) and the fact that $\left(\widetilde{V}_{1}^{*}\right)^{\prime}(-1)=\left(\hat{V}_{1}^{*}\right)^{\prime}(-1)=$ 0 would imply that $\hat{V}_{1}^{*}(0)>\widetilde{V}_{1}^{*}(0)$, a contradiction. Suppose next that the set $\{x \in(-1,0)$ : $\left.\hat{V}_{1}^{*}(x) \geq \widetilde{V}_{1}^{*}(x)\right\}$ is non-empty, and let $z$ be the smallest point in that set. By continuity of $\hat{V}_{1}^{*}$ and $\widetilde{V}_{1}^{*}, \hat{V}_{1}^{*}(z)=\widetilde{V}_{1}^{*}(z)$. Since $\hat{V}_{1}^{*}(x)<\widetilde{V}_{1}^{*}(x)$ for all $x \in[-1, z)$, it must be that $\left(\hat{V}_{1}^{*}\right)^{\prime}(z)>\left(\widetilde{V}_{1}^{*}\right)^{\prime}(z)$. Lemma A2 (i) then implies that $\hat{V}_{1}^{*}(x)>\widetilde{V}_{1}^{*}(x)$ for all $x \in(z, 0]$, a contradiction. Hence, $\widetilde{V}_{1}^{*}(x)>\hat{V}_{1}^{*}(x) \forall x \in[-1,0)$. Therefore, there must exist $x^{*} \geq 0$ such that $\widetilde{V}_{1}^{*}(x) \geq \hat{V}_{1}^{*}(x)$ for all $x \leq x^{*}$, with strict inequality for $x<x^{*}$.

Suppose that $x^{*} \in[0,1)$. I now show that $\widetilde{V}_{1}^{*}(x)<\hat{V}_{1}^{*}(x)$ for all $x>x^{*}$. Note first that $\left(\hat{V}_{1}^{*}\right)^{\prime}\left(x^{*}\right)>\left(\widetilde{V}_{1}\right)^{\prime *}\left(x^{*}\right)$. Towards a contradiction, suppose that there exists $y>x^{*}$ with $\widetilde{V}_{1}^{*}(y) \geq \hat{V}_{1}^{*}(y)$, and let $x^{\prime}=\inf \left\{y>x^{*}: \widetilde{V}_{1}^{*}(y) \geq \hat{V}_{1}^{*}(y)\right\}$. By continuity, $\widetilde{V}_{1}^{*}\left(x^{\prime}\right)=\hat{V}_{1}^{*}\left(x^{\prime}\right)$. Then, by Theorem 1, $\widetilde{V}_{1}^{*}$ and $\hat{V}_{1}^{*}$ both solve ODE $r V(x)=r+\mu V^{\prime}(x)+\frac{1}{2} \sigma^{2} V^{\prime \prime}(x)$ on $x \in\left(x^{*}, x^{\prime}\right)$, with boundary conditions $\widetilde{V}_{1}^{*}\left(x^{*}\right)=\hat{V}_{1}^{*}\left(x^{*}\right)$ and $\widetilde{V}_{1}^{*}\left(x^{\prime}\right)=\hat{V}_{1}^{*}\left(x^{\prime}\right)$. Since this ODE has a unique solution, it must be that $\widetilde{V}_{1}^{*}(x)=\hat{V}_{1}^{*}(x)$ for all $x \in\left(x^{*}, x^{\prime}\right)$. But this cannot be, since $\left(\hat{V}_{1}^{*}\right)^{\prime}\left(x^{*}\right)>\left(\widetilde{V}_{1}\right)^{\prime *}\left(x^{*}\right)$. Hence, it must be that $\widetilde{V}_{1}^{*}(x)<\hat{V}_{1}^{*}(x)$ for all $x>x^{*}$.

The proof that there exists $x^{*} \leq 0$ such that $V_{2}^{*}(x)$ is increasing in $\sigma$ for all $x \in\left[x^{*}, 1\right]$ and decreasing in $\sigma$ for all $x \in\left[-1, x^{*}\right]$ when $\mu \geq 0$ follows from a symmetric argument (but 
using part (ii) of Lemma A2 instead of part (i)).

Proof of Proposition 3. Since $V_{1}^{*}(x)+V_{2}^{*}(x)=1$ for all $x$, to prove Proposition 3 it suffices to show that $V_{1}^{*}$ is increasing in $\mu$ for all $x \in[-1,0]$ and that $V_{2}^{*}$ is decreasing in $\mu$ for all $x \in[0,1]$. Using equation (A.6), it can be shown that

$$
\frac{\partial V_{1}^{*}(0)}{\partial \mu}=\frac{\left(-1+\exp ^{\frac{4 \sqrt{\mu^{2}+2 r \sigma^{2}}}{\sigma^{2}}}\right) r \sigma^{4}+2 \exp ^{\frac{2 \sqrt{\mu^{2}+2 r \sigma^{2}}}{\sigma^{2}}} \mu^{2} \sqrt{\mu^{2}+2 r \sigma^{2}}}{\left(1+\exp ^{\frac{2 \sqrt{\mu^{2}+2 r \sigma^{2}}}{\sigma^{2}}}\right)^{2} \sigma^{2}\left(\mu^{2}+2 r \sigma^{2}\right)^{3 / 2}}>0
$$

Fix $\hat{\mu}<\widetilde{\mu}$ and let $\hat{V}_{1}^{*}$ and $\widetilde{V}_{1}^{*}$ denote player 1's payoff when the drift of $x_{t}$ is $\hat{\mu}$ and $\widetilde{\mu}$, respectively. By the inequality above, $\hat{V}_{1}^{*}(0)<\widetilde{V}_{1}^{*}(0)$. By Theorem $1, \hat{V}_{1}^{*}$ and $\widetilde{V}_{1}^{*}$ solve equation (A.1) on $[-1,0]$ (but with different values of drifts), with $\left(\widetilde{V}_{1}^{*}\right)^{\prime}(-1)=\left(\hat{V}_{1}^{*}\right)^{\prime}(-1)=$ 0 . Moreover, by (A.6), $\hat{V}_{1}^{*}$ and $\widetilde{V}_{1}^{*}$ are strictly increasing and convex for all $x<0$.

I now show that $\widetilde{V}_{1}^{*}(x)>\hat{V}_{1}^{*}(x) \forall x[-1,0]$. Note first that it must be that $\widetilde{V}_{1}^{*}(-1)>$ $\hat{V}_{1}^{*}(-1)$ : if $\hat{V}_{1}^{*}(-1) \geq \widetilde{V}_{1}^{*}(-1)$, then Lemma A2 (i) and the fact that $\left(\widetilde{V}_{1}^{*}\right)^{\prime}(-1)=\left(\hat{V}_{1}^{*}\right)^{\prime}(-1)=$ 0 would imply that $\hat{V}_{1}^{*}(0)>\widetilde{V}_{1}^{*}(0)$, a contradiction. Let $z>0$ be the smallest point such that $\hat{V}_{1}^{*}(z)=\widetilde{V}_{1}^{*}(z)$, and suppose by contradiction that $z<0$. Since $\widetilde{V}_{1}^{*}(x)>\hat{V}_{1}^{*}(x)$ for all $x<z$, it must be that $\left(\hat{V}_{1}^{*}\right)^{\prime}(z)>\left(\widetilde{V}_{1}^{*}\right)^{\prime}(z)$. Lemma A2 (i) then implies that $\hat{V}_{1}^{*}(x)>\widetilde{V}_{1}^{*}(x)$ for all $x \in(z, 0]$, which contradicts the fact that $\widetilde{V}_{1}^{*}(0)>\hat{V}_{1}^{*}(0)$. Hence, $\widetilde{V}_{1}^{*}(x)>\hat{V}_{1}^{*}(x)$ $\forall x \in[-1,0]$. The proof that $V_{2}^{*}$ is decreasing in $\mu$ for $x \in[0,1]$ follows from a symmetric argument (but using part (ii) of Lemma A2 instead of part (i)).

\section{A.2 Proofs of Section 3.2}

Consider the model in section 3.2. As before, an outcome is a pair $(A, \eta)$, where $A$ is the agreement region and $\eta: A \rightarrow[0,1]$ a policy function. Party $i$ 's payoff from outcome $(A, \eta)$ is

$$
V_{i}(x)=\mathbb{E}\left[e^{-r \tau(A)} u_{i}\left(\eta\left(x_{\tau(A)}\right)\right) \mid x_{0}=x\right],
$$


where $\tau(A):=\inf \left\{t: x_{t} \in A\right\}$.

Under supermajority rule $\phi \leq 1$, an equilibrium outcome $(A, \eta)$ must satisfy the following conditions: (i) $A \supseteq[-1,-\phi] \cup[\phi, 1]$, (ii) $\eta(x)=0$ for all $x \in[\phi, 1]$, and (iii) $\eta(x)=1$ for all $x \in[-1,-\phi]$. Indeed, a party with a supermajority always finds it strictly optimal to implement its preferred policy immediately and earn a payoff of 1 . For each $\phi \in(0,1]$, let $\mathcal{O}^{\phi}$ denote the set of outcomes satisfying conditions (i)-(iii).

Recall that $T_{1}$ is the set of stopping times $\tau$ such that $x_{\tau} \in[0,1]$, and that $T_{2}$ is the set of stopping times $\tau$ such that $x_{\tau} \in[-1,0]$. Let $\tau^{\phi}=\inf \left\{t: x_{t} \notin(-\phi, \phi)\right\}$ be the first time a party obtains a supermajority. The following definition adapts Definition 1 to this model.

Definition 3 Under supermajority rule $\phi$ an outcome $\left(A, V_{1}, V_{2}\right) \in \mathcal{O}^{\phi}$ is an equilibrium if, for $i, j=1,2, j \neq i$,

$$
V_{i}(x)=\sup _{\tau \in T_{i}} \mathbb{E}\left[e^{-r \min \left\{\tau, \tau^{\phi}\right\}}\left(1-V_{j}\left(x_{\min \left\{\tau, \tau^{\phi}\right\}}\right)\right) \mid x_{0}=x\right] \forall x \in[-1,1]
$$

Theorem A1 For any $\phi \in(0,1]$ there is a unique equilibrium $\left(A^{\phi}, V_{1}^{\phi}, V_{2}^{\phi}\right)$. The equilibrium agreement region is $A^{\phi}=[-1,1]$. For all $x \in(-\phi, \phi)$, equilibrium payoffs $V_{i}^{\phi}(x)$ solve

$$
r V_{i}^{\phi}(x)= \begin{cases}\mu\left(V_{i}^{\phi}\right)^{\prime}(x)+\frac{1}{2} \sigma^{2}\left(V_{i}^{\phi}\right)^{\prime \prime}(x) & \text { if } x \notin A_{i}^{*}, \\ r+\mu\left(V_{i}^{\phi}\right)^{\prime}(x)+\frac{1}{2} \sigma^{2}\left(V_{i}^{\phi}\right)^{\prime \prime}(x) & \text { if } x \in A_{i}^{*},\end{cases}
$$

with $V_{1}^{*}(-\phi)=V_{2}^{*}(\phi)=0, V_{1}^{*}(\phi)=V_{2}^{*}(-\phi)=1, \lim _{x \uparrow 0} V_{i}^{\phi}(x)=\lim _{x \downarrow 0} V_{i}^{\phi}(x)$ and $\lim _{x \uparrow 0}\left(V_{i}^{\phi}\right)^{\prime}(x)=\lim _{x \downarrow 0}\left(V_{i}^{\phi}\right)^{\prime}(x)$.

Proof. By the same arguments as in Proposition 1, any equilibrium $\left(A, V_{1}, V_{2}\right)$ has $A=A^{\phi}=$ $[-1,1]$. Recall that $A_{1}^{*}=[0,1]$ and $A_{2}^{*}=[-1,0]$. For $i=1,2$, let $\tau\left(A_{i}^{*}\right)=\inf \left\{t: x_{t} \in A_{i}^{*}\right\}$. 
Let $\tau^{\phi}=\inf \left\{t: x_{t} \notin(-\phi, \phi)\right\}$. Using equation (A.12), for all $x \notin A_{i}^{*}, V_{i}(x)$ satisfies

$$
\begin{aligned}
V_{i}(x) & =\mathbb{E}\left[e^{-r \min \left\{\tau\left(A_{i}^{*}\right), \tau^{\phi}\right\}}\left(1-V_{j}\left(x_{\min \left\{\tau\left(A_{i}^{*}\right), \tau^{\phi}\right\}}\right)\right) \mid x_{0}=x\right] \\
& =\mathbb{E}\left[e^{-r \min \left\{\tau(0), \tau^{\phi}\right\}}\left(1-V_{j}\left(x_{\min \left\{\tau(0), \tau^{\phi}\right\}}\right)\right) \mid x_{0}=x\right]
\end{aligned}
$$

with $V_{1}(\phi)=V_{2}(-\phi)=1, V_{1}(-\phi)=V_{2}(\phi)=0$ and $V_{i}(0)=1-V_{j}(0)$ (the equality in the equation follows since $\tau\left(A_{i}^{*}\right)=\tau(0)$ whenever $x_{0} \notin A_{i}^{*}$ ). Note that, for all $x \notin A_{1}^{*}$ (i.e., for all $x \in[-1,0]), \min \left\{\tau(0), \tau^{\phi}\right\}=\tau_{-\phi 0}=\inf \left\{t: x_{t} \notin(-\phi, 0)\right\}$. Similarly, for all $x \notin A_{2}^{*}$ (i.e., $x \in[0,1]), \min \left\{\tau(0), \tau^{\phi}\right\}=\tau_{0 \phi}=\inf \left\{t: x_{t} \notin(0, \phi)\right\}$. By Lemma A1(iii), for all $x \in[-\phi, 0]$, $V_{1}(x)$ solves

$$
r V_{1}(x)=\mu V_{1}^{\prime}(x)+\frac{1}{2} \sigma^{2} V_{1}^{\prime \prime}(x),
$$

with boundary conditions $V_{1}(-\phi)=0$ and $V_{1}(0)=1-V_{2}(0)$. Similarly, for all $x \in[0, \phi]$, $V_{2}(x)$ solves

$$
r V_{2}(x)=\mu V_{2}^{\prime}(x)+\frac{1}{2} \sigma^{2} V_{2}^{\prime \prime}(x),
$$

with boundary conditions $V_{2}(\phi)=0$ and $V_{2}(0)=1-V_{1}(0)$.

Let $A_{1}^{\phi}:=[0, \phi]$ and $A_{2}^{\phi}[-\phi, 0]$. Since $V_{i}(x)=1-V_{j}(x) \forall x$, the equations above imply

$$
r V_{i}(x)= \begin{cases}\mu V_{i}^{\prime}(x)+\frac{1}{2} \sigma^{2} V_{i}^{\prime \prime}(x) & \text { if } x \in A_{j}^{\phi}, \\ r+\mu V_{i}^{\prime}(x)+\frac{1}{2} \sigma^{2} V_{i}^{\prime \prime}(x) & \text { if } x \in A_{i}^{\phi},\end{cases}
$$

with boundary conditions $V_{1}(\phi)=V_{2}(-\phi)=1, V_{1}(-\phi)=V_{2}(\phi)=0$ and $\lim _{x \uparrow 0} V_{i}(x)=$ $\lim _{x \downarrow 0} V_{i}(x)$. The same arguments as in the proof of Theorem 1 can be used to show that any equilibrium must be such that $\lim _{x \uparrow 0} V_{i}^{\prime}(x)=\lim _{x \downarrow 0} V_{i}^{\prime}(x)$.

Proof of Proposition 4. For $i=1,2$, let $V_{i}^{\tilde{\phi}}(\cdot)$ be party $i$ 's payoff under supermajority $\tilde{\phi}$. Note that $V_{1}^{\phi^{\prime}}(-\phi)>V_{1}^{\phi}(-\phi)=0$ and $V_{1}^{\phi^{\prime}}\left(\phi^{\prime}\right)<V_{1}^{\phi}(\phi)=1$. Let $x_{1}:=\inf \{x \in[-\phi, \phi]$ : $\left.V_{1}^{\phi^{\prime}}(x) \leq V_{1}^{\phi}(x)\right\}$ and $x_{2}:=\sup \left\{x \in[-\phi, \phi]: V_{1}^{\phi^{\prime}}(x) \geq V_{1}^{\phi}(x)\right\}$. To prove the proposition, it suffices to show that $x_{1}=x_{2}$. 
Suppose first that $x_{1} \leq 0$. Note that $V_{1}^{\phi^{\prime}}\left(x_{1}\right)=V_{1}^{\phi}\left(x_{1}\right)$ and $\left(V_{1}^{\phi^{\prime}}\right)^{\prime}\left(x_{1}\right)<\left(V_{1}^{\phi}\right)^{\prime}\left(x_{1}\right)$. Since $V_{1}^{\phi^{\prime}}(\cdot)$ and $V_{1}^{\phi}(\cdot)$ both solve (A.1) on $[-\phi, 0]$ (Theorem A1), Lemma A2 implies that $V_{1}^{\phi^{\prime}}(x) \leq V_{1}^{\phi}(x)$ for all $x \in\left[x_{1}, 0\right]$ and $\left(V_{1}^{\phi^{\prime}}\right)^{\prime}(0)<\left(V_{1}^{\phi}\right)^{\prime}(0)$. This in turn implies that $V_{2}^{\phi^{\prime}}(0)=$ $1-V_{1}^{\phi^{\prime}}(0) \geq V_{2}^{\phi}(0)=1-V_{1}^{\phi}(x)$ and that $\left(V_{2}^{\phi^{\prime}}\right)^{\prime}(0)=-\left(V_{1}^{\phi^{\prime}}\right)^{\prime}(0)>\left(V_{2}^{\phi}\right)^{\prime}(0)=-\left(V_{1}^{\phi}\right)^{\prime}(0)$. Since $V_{2}^{\phi^{\prime}}(\cdot)$ and $V_{2}^{\phi}(\cdot)$ both solve (A.1) on $[0, \phi]$, Lemma A2 implies that $V_{2}^{\phi^{\prime}}(x)>V_{2}^{\phi}(x)$ for all $x \in(0, \phi)$. This in turn implies that $V_{1}^{\phi^{\prime}}(x)=1-V_{2}^{\phi^{\prime}}(x)<1-V_{2}^{\phi}(x)=V_{1}^{\phi}(x)$ for all $x \in\left(x_{1}, \phi\right]$, and so $x_{1}=x_{2}$.

Suppose next that $x_{1}>0$. Note that $V_{1}^{\phi^{\prime}}\left(x_{1}\right)=V_{1}^{\phi}\left(x_{1}\right)$ and $\left(V_{1}^{\phi^{\prime}}\right)^{\prime}\left(x_{1}\right)<\left(V_{1}^{\phi}\right)^{\prime}\left(x_{1}\right)$. Hence, $V_{2}^{\phi^{\prime}}\left(x_{1}\right)=1-V_{1}^{\phi^{\prime}}\left(x_{1}\right) \geq V_{2}^{\phi}\left(x_{1}\right)=1-V_{1}^{\phi}\left(x_{1}\right)$ and $\left(V_{2}^{\phi^{\prime}}\right)^{\prime}\left(x_{1}\right)=-\left(V_{1}^{\phi^{\prime}}\right)^{\prime}\left(x_{1}\right)>$ $\left(V_{2}^{\phi}\right)^{\prime}\left(x_{1}\right)=-\left(V_{1}^{\phi}\right)^{\prime}\left(x_{1}\right)$. Since $V_{2}^{\phi^{\prime}}(\cdot)$ and $V_{2}^{\phi}(\cdot)$ both solve (A.1) on $\left[x_{1}, \phi\right]$, Lemma A2 implies that $V_{2}^{\phi^{\prime}}(x)>V_{2}^{\phi}(x)$ for all $x \in\left(x_{1}, \phi\right)$. This in turn implies that $V_{1}^{\phi^{\prime}}(x)=1-V_{2}^{\phi^{\prime}}(x)<$ $1-V_{2}^{\phi}(x)=V_{1}^{\phi}(x)$ for all $x \in\left(x_{1}, \phi\right]$, and so $x_{1}=x_{2}$.

\section{A.3 Proofs of Section 4}

Let $F^{2}$ be the set of bounded and measurable functions on $[-1,1]$ taking values on $\mathbb{R}^{2}$. Let $\|\cdot\|_{2}$ denote the sup norm on $\mathbb{R}^{2}$. For any $f \in F^{2}$, let $\|f\|=\sup _{x \in[-1,1]}\|f(x)\|_{2}$. Fix $\Delta>0$ and $r>0$. Recall that $A_{1}^{*}=[0,1]$ and $A_{2}^{*}=[-1,0]$. Define the operator $\psi: F^{2} \rightarrow F^{2}$ as follows: for any $f=\left(f_{1}, f_{2}\right) \in F^{2}$ and for $i, j=1,2, i \neq j$,

$$
\psi_{i}\left(f_{i}, f_{j}\right)(x)=\left\{\begin{array}{lc}
e^{-r \Delta} \mathbb{E}\left[f_{i}\left(x_{t+\Delta}\right) \mid x_{t}=x\right] & \text { if } x \notin A_{i}^{*}, \\
1-e^{-r \Delta} \mathbb{E}\left[f_{j}\left(x_{t+\Delta}\right) \mid x_{t}=x\right] & \text { if } x \in A_{i}^{*},
\end{array}\right.
$$

Note that $\psi$ is a contraction of modulus $e^{-r \Delta}<1$. Indeed, for any $f, g \in F^{2},\|\psi(f)-\psi(g)\| \leq$ $e^{-r \Delta}\|f-g\|$. Therefore, $\psi$ has a unique fixed point.

Proof of Theorem 2. To prove Theorem 2, I start out assuming that the set of SPE of $\Gamma_{\Delta}$ is non-empty. At the end of the proof I show that $\Gamma_{\Delta}$ has a SPE. Fix a SPE of $\Gamma_{\Delta}$ and let $f_{i}(x)$ be player $i$ 's payoff from this SPE when $x_{0}=x$. Let $\bar{U}=\left(\bar{U}_{1}, \bar{U}_{2}\right) \in F^{2}$ and $\bar{u}=\left(\bar{u}_{1}, \bar{u}_{2}\right) \in F^{2}$ 
be the players' supremum and infimum SPE payoffs, so $f_{i}(x) \in\left[\bar{u}_{i}(x), \bar{U}_{i}(x)\right] \forall x \in[-1,1]$.

Note that for all $x \in A_{i}^{*}$ player $i$ 's SPE payoff is bounded below by $1-e^{-r \Delta} \mathbb{E}\left[\bar{U}_{j}\left(x_{t+\Delta}\right) \mid x_{t}=\right.$ $x]$, since in any SPE player $j$ always accepts an offer that gives her a payoff equal to $e^{-r \Delta} \mathbb{E}\left[\bar{U}_{j}\left(x_{t+\Delta}\right) \mid x_{t}=x\right]$. On the other hand, for all $x \notin A_{i}^{*}$ player $i$ 's payoff is bounded below by $e^{-r \Delta} \mathbb{E}\left[\bar{u}_{i}\left(x_{t+\Delta}\right) \mid x_{t}=x\right]$, since player $i$ can always guarantee this payoff by rejecting party $j$ 's offer. Thus, for all $x \in[-1,1]$ it must be that $f_{i}(x) \geq \bar{u}_{i}(x) \geq \psi_{i}\left(\bar{u}_{i}, \bar{U}_{j}\right)(x)$.

At states $x \notin A_{i}^{*}$, player $i$ 's payoff is bounded above by $e^{-r \Delta} \mathbb{E}\left[\bar{U}_{i}\left(x_{t+\Delta}\right) \mid x_{t}=x\right]$, since player $j$ will never make an offer that gives player $i$ a payoff larger than this. Consider next states $x \in A_{i}^{*}$, and note that $f_{i}(x)+f_{j}(x) \leq 1$. This inequality follows since the sum of the players SPE payoffs cannot be larger than what they get by agreeing immediately. Moreover, by the arguments in the previous paragraph, $f_{j}(x) \geq e^{-r \Delta} \mathbb{E}\left[\bar{u}_{j}\left(x_{t+\Delta}\right) \mid x_{t}=x\right]$ for all $x \in A_{i}^{*}$. These two inequalities imply that $f_{i}(x) \leq 1-e^{-r \Delta} \mathbb{E}\left[\bar{u}_{j}\left(x_{t+\Delta}\right) \mid x_{t}=x\right]$ for all $x \in A_{i}^{*}$. Thus, $f_{i}(x) \leq \bar{U}_{i}(x) \leq \psi_{i}\left(\bar{U}_{i}, \bar{u}_{j}\right)(x) \forall x \in[-1,1]$.

The two paragraphs above imply that, for $i=1,2, i \neq j$, and for all $x \in[-1,1]$,

$$
\begin{aligned}
\bar{U}_{i}(x)-\bar{u}_{i}(x) & \leq \psi_{i}\left(\bar{U}_{i}, \bar{u}_{j}\right)(x)-\psi_{i}\left(\bar{u}_{i}, \bar{U}_{j}\right)(x) \\
& \leq \max _{k \in\{1,2\}} e^{-r \Delta} \mathbb{E}\left[\bar{U}_{k}\left(x_{t+\Delta}\right)-\bar{u}_{k}\left(x_{t+\Delta}\right) \mid x_{t}=x\right],
\end{aligned}
$$

where the last inequality follows from the definition of $\psi_{i}$. Since the inequality above holds for $i=1,2$ and for all $x \in[-1,1],\|\bar{U}-\bar{u}\| \leq e^{-r \Delta}\|\bar{U}-\bar{u}\|$. Hence, $\bar{U}=\bar{u}$ and SPE payoffs are unique. Let $V^{\Delta}=\left(V_{1}^{\Delta}, V_{2}^{\Delta}\right) \in F^{2}$ be the unique SPE payoffs. Since $\psi_{i}\left(\bar{U}_{i}, \bar{u}_{j}\right) \geq V_{i}^{\Delta} \geq$ $\psi_{i}\left(\bar{u}_{i}, \bar{U}_{j}\right)$ and since $\bar{U}=\bar{u}$, it follows that $V^{\Delta}$ is the unique fixed point of $\psi$. Note that $V_{1}^{\Delta}$ and $V_{2}^{\Delta}$ and satisfy equation (5) in Theorem 2 .

The arguments above show that all $\mathrm{SPE}$ of $\Gamma_{\Delta}$ are payoff equivalent. I now show that there exists a unique SPE. Since $V_{1}^{\Delta}(x)+V_{2}^{\Delta}(x)=1$ for all $x \in[-1,1]$, in any SPE players always reach an immediate agreement, and their payoffs from this agreement are $\left(V_{1}^{\Delta}\left(x_{0}\right), V_{2}^{\Delta}\left(x_{0}\right)\right)$. I now use this to construct the unique SPE. Consider the following strategy profile. At every 
$x \in A_{i}^{*}$, player $i$ makes an offer that gives players a payoff equal to $\left(V_{1}^{\Delta}(x), V_{2}^{\Delta}(x)\right)$, and player $j \neq i$ only accepts offers that give her a payoff at least as large as $V_{j}^{\Delta}(x)$. The players' payoffs from this strategy profile are $\left(V_{1}^{\Delta}, V_{2}^{\Delta}\right)$. Moreover, it is easy to see that no player can gain by deviating from its strategy at any $x \in[-1,1]$. Hence, this strategy profile is a SPE of $\Gamma_{\Delta}$.

Proof of Theorem 3. By equation (5) in Theorem 2, player $i$ 's payoff for all $x \in A_{i}^{*}$ satisfies

$$
V_{i}^{\Delta}(x)=1-e^{-r \Delta} \mathbb{E}\left[V_{j}^{\Delta}\left(x_{t+\Delta}\right) \mid x_{t}=x\right]=1-e^{-r \Delta}+e^{-r \Delta} \mathbb{E}\left[V_{i}^{\Delta}\left(x_{t+\Delta}\right) \mid x_{t}=x\right],
$$

where the second equality follows since $V_{1}^{\Delta}(x)+V_{2}^{\Delta}(x)=1$ for all $x \in[-1,1]$. Since $V_{i}^{\Delta}(x)=e^{-r \Delta} \mathbb{E}\left[V_{i}^{\Delta}\left(x_{t+\Delta}\right) \mid x_{t}=x\right]$ for all $x \notin A_{i}^{*}$, it follows that

$$
V_{i}^{\Delta}(x)=\left(1-e^{-r \Delta}\right) \mathbf{1}_{\left\{x \in A_{i}^{*}\right\}}+e^{-r \Delta} \mathbb{E}\left[V_{i}^{\Delta}\left(x_{t+\Delta}\right) \mid x_{t}=x\right] .
$$

Setting $t=0$ and solving equation (A.15) forward yields

$$
V_{i}^{\Delta}(x)=\mathbb{E}\left[\left(1-e^{-r \Delta}\right) \sum_{k=0}^{\infty} e^{-r k \Delta} \mathbf{1}_{\left\{x_{k \Delta} \in A_{i}^{*}\right\}} \mid x_{0}=x\right]=\frac{1-e^{-r \Delta}}{\Delta} \sum_{k=0}^{\infty} \Delta e^{-r k \Delta} P_{i}(\Delta k, x),
$$

where, for all $s \geq 0$ and all $x \in[-1,1], P_{i}(s, x):=\mathbb{E}\left[\mathbf{1}_{\left\{x_{s} \in A_{i}^{*}\right\}} \mid x_{0}=x\right]$ is the probability with which player $i$ has proposal power at time $s$ conditional on $x_{0}=x$.

For all $s>0$ and all $x, y \in[0,1]$, let $p(x, y, s)=\operatorname{Prob}\left(x_{s}=y \mid x_{0}=x\right)$ be the transition density function of the process $x_{t}$. It is well known that $p(x, y, s)$ solves Kolmogorov's backward equation (e.g., Bhattacharya and Waymire (2009), chapter V.6),

$$
\frac{\partial}{\partial s} p(x, y, s)=\mu \frac{\partial}{\partial x} p(x, y, s)+\frac{1}{2} \sigma^{2} \frac{\partial^{2}}{\partial x^{2}} p(x, y, s)
$$

with $\lim _{s \downarrow 0} p(x, y, s)=\mathbf{1}_{\{y=x\}}$ and $\left.\frac{\partial}{\partial x} p(x, y, s)\right|_{x=-1}=\left.\frac{\partial}{\partial x} p(x, y, s)\right|_{x=1}=0$ for all $s>0$. Note 
that for all $s>0$ and for $i=1,2, P_{i}(s, x)=\mathbb{E}\left[\mathbf{1}_{\left\{x_{s} \in A_{i}^{*}\right\}} \mid x_{0}=x\right]=\int_{A_{i}^{*}} p(x, y, s) d y$. Since $p(x, y, s)$ solves (A.17) with $\left.\frac{\partial}{\partial x} p(x, y, s)\right|_{x=-1}=\left.\frac{\partial}{\partial x} p(x, y, s)\right|_{x=1}=0$ and $\lim _{s \downarrow 0} p(x, y, s)=$ $\mathbf{1}_{\{y=x\}}$, it follows that $P_{i}(s, x)$ also solves (A.17), with $\lim _{s \downarrow} P_{i}(s, x)=P_{i}(0, x)=\mathbf{1}_{\left\{x \in A_{i}^{*}\right\}}$ and $\left.\frac{\partial}{\partial x} P_{i}(s, x)\right|_{x=-1}=\left.\frac{\partial}{\partial x} P_{i}(s, x)\right|_{x=1}=0$ for all $s \geq 0 .{ }^{12} \quad$ Note that $P_{i}(\cdot, x)$ is continuous on $(0, \infty)$ (being differentiable). Hence, $e^{-r s} P_{i}(s, x)$ is Riemann integrable, and so $\sum_{k=0}^{\infty} \Delta e^{-r k \Delta} P_{i}(\Delta k, x) \rightarrow \int_{0}^{\infty} e^{-r t} P(t, x) d t$ as $\Delta \rightarrow 0$. It then follows from (A.16) that $V_{i}^{\Delta}(x)$ converges pointwise to $W_{i}(x):=r \int_{0}^{\infty} e^{-r t} P(t, x) d t$ as $\Delta \rightarrow 0$.

I use the following Lemma:

Lemma A3 For $i=1,2, W_{i}(\cdot)$ is continuous.

Proof. For $i=1,2$ and for every $\varepsilon>0$, let $V_{i}^{\varepsilon}(x):=r \int_{\varepsilon}^{\infty} e^{-r t} P_{i}(t, x) d t$. Since $P_{i}(t, \cdot)$ is continuous for all $t>0$ (being differentiable), $V_{i}^{\varepsilon}(\cdot)$ is continuous for all $\varepsilon>0$. To show that $W_{i}(\cdot)$ is continuous, it suffices to show that $V_{i}^{\varepsilon}(x) \rightarrow W_{i}(x)$ uniformly as $\varepsilon \rightarrow 0$. For any $\varepsilon>0$ and any $x \in[-1,1],\left|W_{i}(x)-V_{i}^{\varepsilon}(x)\right|=r \int_{0}^{\varepsilon} e^{-r t} P_{i}(t, x) d t \leq r \int_{0}^{\varepsilon} e^{-r t} d t=1-e^{-r \varepsilon}$ (since $\left.P_{i}(t, x) \in[0,1]\right)$. Since $\lim _{\varepsilon \rightarrow 0} 1-e^{-r \varepsilon}=0, V_{i}^{\varepsilon}(\cdot) \rightarrow W_{i}(\cdot)$ uniformly as $\varepsilon \rightarrow 0$.

Note that, for all $t>0, P_{1}(t, x)$ is increasing in $x$, since player 1 is more likely to have proposal power at $t>0$ if $x_{0}=x$ is larger. Similarly, $P_{2}(t, x)$ is decreasing in $x$. Therefore, by (A.16), for all $\Delta>0 V_{i}^{\Delta}(\cdot)$ is monotone on $[-1,1]$. Since $W_{i}(\cdot)$ is continuous, it follows that $V_{i}^{\Delta}(\cdot) \rightarrow W_{i}(\cdot)$ uniformly as $\Delta \rightarrow 0$.

I now complete the proof of Theorem 3. By the arguments above, $V_{i}^{\Delta}(x) \rightarrow W_{i}(x)=$ $r \int_{0}^{\infty} e^{-r t} P(t, x) d t$ uniformly as $\Delta \rightarrow 0$. Integrating by parts, for all $x \neq 0$

$$
W_{i}(x)=r \int_{0}^{\infty} e^{-r t} P_{i}(t, x) d t=-\left.e^{-r t} P_{i}(t, x)\right|_{0} ^{\infty}+\int_{0}^{\infty} e^{-r t} \frac{\partial}{\partial t} P_{i}(t, x) d t
$$

Note that $-\left.e^{-r t} P_{i}(t, x)\right|_{0} ^{\infty}=P_{i}(0, x)=\mathbf{1}_{\left\{x \in A_{i}^{*}\right\}}$. Hence, $\forall x \neq 0, W_{i}(x)=\mathbf{1}_{\left\{x \in A_{i}^{*}\right\}}+$

\footnotetext{
${ }^{12}$ Since $p(x, y, s)$ satisfies (A.17), for all $x \in[-1,1]$ and all $s>0, \frac{\partial}{\partial s} P_{i}(s, x)=\int_{A_{i}^{*}} \frac{\partial}{\partial s} p(x, y, s) d y=$ $\mu \int_{A_{i}^{*}} \frac{\partial}{\partial x} p(x, y, s) d y+\frac{1}{2} \sigma^{2} \int_{A_{i}^{*}} \frac{\partial^{2}}{\partial x^{2}} p(x, y, s) d y=\mu \frac{\partial}{\partial x} P_{i}(s, x)+\frac{1}{2} \sigma^{2} \frac{\partial^{2}}{\partial x^{2}} P_{i}(s, x)$.
} 
$\int_{0}^{\infty} e^{-r t} \frac{\partial}{\partial t} P_{i}(t, x) d t$. Since $\frac{\partial}{\partial t} P_{i}(t, x)=\mu \frac{\partial}{\partial x} P_{i}(t, x)+\frac{\sigma^{2}}{2} \frac{\partial^{2}}{\partial x^{2}} P_{i}(t, x)$ for all $t>0$ (see footnote (12)), it follows that for all $x \neq 0$,

$$
\begin{aligned}
r W_{i}(x) & =r \times \mathbf{1}_{\left\{x \in A_{i}^{*}\right\}}+r \int_{0}^{\infty} e^{-r t}\left(\mu \frac{\partial}{\partial x} P_{i}(t, x)+\frac{1}{2} \sigma^{2} \frac{\partial^{2}}{\partial x^{2}} P_{i}(t, x)\right) d t \\
& =r \times \mathbf{1}_{\left\{x \in A_{i}^{*}\right\}}+\mu W_{i}^{\prime}(x)+\frac{1}{2} \sigma^{2} W_{i}^{\prime \prime}(x),
\end{aligned}
$$

where the second equality follows since $W_{i}^{\prime}(x)=r \int_{0}^{\infty} e^{-r t} \frac{\partial}{\partial x} P_{i}(t, x) d t$ and since $W_{i}^{\prime \prime}(x)=$ $r \int_{0}^{\infty} e^{-r t} \frac{\partial^{2}}{\partial x^{2}} P_{i}(t, x) d t$. Therefore, $W_{i}^{*}(\cdot)$ solves the same differential equation than $V_{i}^{*}(\cdot)$ for all $x \neq 0$. To pin down the boundary conditions, note that $W_{i}^{\prime}(x)=r \int_{0}^{\infty} e^{-r t} \frac{\partial}{\partial x} P_{i}(t, x) d t$. Since $\left.\frac{\partial}{\partial x} P_{i}(t, x)\right|_{x=-1}=\left.\frac{\partial}{\partial x} P_{i}(t, x)\right|_{x=1}=0$ for all $t \geq 0$, it follows that $W_{i}^{\prime}(-1)=W_{i}^{\prime}(1)=0$.

Since $W_{i}(\cdot)$ is continuous (Lemma A3), it must be that $\lim _{x \uparrow 0} W_{i}(x)=\lim _{x \downarrow 0} W_{i}(x)$. Finally, integrating both sides of (A.18) yields

$$
\int_{-1}^{z} r W_{i}(x) d x=\int_{-1}^{z} r \mathbf{1}_{\left\{x \in A_{i}^{*}\right\}} d x+\mu\left(W_{i}(z)-W_{i}(-1)\right)+\frac{1}{2} \sigma^{2}\left(\left(W_{i}\right)^{\prime}(z)-\left(W_{i}\right)^{\prime}(-1)\right) .
$$

Note that the two integrals in the equation above are continuous in $z$. Since $W_{i}(\cdot)$ is also continuous, $W_{i}^{\prime}(\cdot)$ must be continuous as well, and so $\lim _{x \uparrow 0} W_{i}^{\prime}(x)=\lim _{x \downarrow 0} W_{i}^{\prime}(x)$.

The paragraphs above imply that $W_{i}(\cdot)$ and $V_{i}^{*}(\cdot)$ both solve the same differential equation, with the same boundary conditions. Since this differential equation has a unique solution, $W_{i}(\cdot)=V_{i}^{*}(\cdot)$.

\section{References}

Ambrus, A. AND S. E. Lu (2015): "A continuous-time model of multilateral bargaining," American Economic Journal: Microeconomics, 7, 208-249.

Bergin, J. AND W. B. MacLeod (1993): "Continuous time repeated games," International Economic Review, 21-37. 
Bhattacharya, R. N. And E. C. WAymire (2009): Stochastic processes with applications, vol. 61 , Siam.

Daley, B. And B. S. Green (2018): "Bargaining and news," .

Harrison, J. M. (1985): Brownian motion and stochastic flow systems, Wiley New York.

KallenberG, O. (2006): Foundations of modern probability, Springer Science \& Business Media.

OrTner, J. (2017a): "Durable goods monopoly with stochastic costs," Theoretical Economics, 12, 817-861.

- (2017b): "A theory of political gridlock," Theoretical Economics, 12, 555-586.

Perry, M. AND P. J. RENy (1993): "A non-cooperative bargaining model with strategically timed offers," Journal of Economic Theory, 59, 50-77.

Rubinstein, A. (1982): "Perfect equilibrium in a bargaining model," Econometrica: Journal of the Econometric Society, 97-109.

SAKOVICS, J. (1993): "Delay in bargaining games with complete information," Journal of Economic Theory, 59, 78-95.

Shiryaev, A. N. (2007): Optimal stopping rules, vol. 8, Springer Science \& Business Media.

Simon, L. K. And M. B. Stinchcombe (1989): "Extensive form games in continuous time: Pure strategies," Econometrica: Journal of the Econometric Society, 1171-1214.

SimseK, A. AND M. YILDIZ (2014): "Durability, deadline, and election effects in bargaining," Unpublished manuscript.

Villamizar, I. C. (2018): "Privacy in Bargaining: The Case of Endogenous Entry," . 